\title{
Original
}

\section{Cisplatin-Induced Sonic Hedgehog Signaling Mediates Epithelial-Mesenchymal Transition in Hertwig's Epithelial Root Sheath Cells}

\author{
Hanako Ishii $^{1,2)}$, Mizuki Yoshida ${ }^{2,3)}$, Hiroshi Kajiya ${ }^{2,4)}$, Satoru Matsuo ${ }^{1)}$, Masako Toda-Nakamura ${ }^{1)}$, Nana Mori-Yamamoto ${ }^{2,5)}$, \\ Seiichi Fujisaki ${ }^{2,6)}$, Kyoko Oka ${ }^{1)}$, Masao Ozaki ${ }^{1)}$ and Jun Ohno ${ }^{2)}$ \\ 1) Section of Pediatric, Department of Oral Growth and Development, Fukuoka Dental College, Fukuoka, Japan \\ 2) Oral Medicine Research Center, Fukuoka Dental College, Fukuoka, Japan \\ 3) Section of Geriatric Dentistry, Department of General Dentistry, Fukuoka Dental College, Fukuoka, Japan \\ 4) Section of Cellular Physiology, Department of Physiological Science and Molecular Biology, Fukuoka Dental College, Fukuoka, Japan \\ ${ }^{5)}$ Section of Periodontology, Department of Odontology, Fukuoka Dental College, Fukuoka, Japan \\ 6) Section of Oral Implantology, Department of Oral Rehabilitation, Fukuoka Dental College, Fukuoka, Japan \\ (Accepted for publication, January 18, 2021)
}

\begin{abstract}
In this study, we determined whether cisplatin can induce epithelial-mesenchymal transition (EMT) via the activation of Sonic hedgehog (Shh) or glioma-associated antigen-1 (Gli1) signaling pathway in mouse Hertwig's epithelial root sheath (HERS) cells using a genetic knockdown approach. HERS cells treated with a low concentration of cisplatin $(0.5$ $\mu \mathrm{M}$ ) for $24 \mathrm{~h}$ showed no reduction in the cell viability; however, there was a significant increase in the percentages of nuclear staining with $\gamma \mathrm{H} 2 \mathrm{AX}$ as compared to that with untreated control cells, indicating that $0.5 \mu \mathrm{M}$ cisplatin induces DNA damage. Further, $0.5-\mu \mathrm{M}$ cisplatin-treated cells provided an induction of EMT, showing decreased and increased expression of epithelial and mesenchymal markers, respectively. Enhancement in the EMT activity in cisplatin-treated HERS cells was correlated with increased expression of Shh and accelerated translocation and accumulation of Glil expression into the nucleus. The RNA interference-mediated silencing of Gli1 suppressed the acceleration of EMT in cisplatin-treated HERS cells; this was confirmed by no down-regulation or up-regelation in the expression of E-cadherin and vimentin, respectively, along with no increased expression of Snail expression. These findings suggest that the activation of Shh/Gli1 signaling pathway may be required for the enhancement of EMT in cisplatin-treated HERS cells.
\end{abstract}

Key words: Hertwig's epithelial root sheath (HERS), Epithelial-mesenchymal transition (EMT), Cisplatin, Sonic hedgehog, Glioma-associated antigen-1, E-cadherin, Vimentin, Snail

\section{Introduction}

Chemotherapeutic drugs are widely used and are effective in the treatment of pediatric cancers. Among many chemotherapeutic drugs, cisplatin, one of major platinum compounds, currently serve as the first line chemotherapy in the treatment of pediatric cancers, such as neuroblastoma, germ-cell tumors, osteosarcoma, retinoblastoma, hepatoblastoma, brain tumors, and relapsed and refractory lymphomas ${ }^{1)}$. Cisplatin forms DNA-protein cross-links, causing breakage of DNA strands in cancer cells, to exert cytotoxic effects and trigger apoptosis in these $\mathrm{cells}^{2}$. Chemotherapy-induced DNA damage may provide some palliation; however, it is often associated with substantial treatment-related toxic effects.

Recent strong evidence supports an association between chemotherapy and dental anomalies. In particular, children who receive chemotherapy before the age of $5 \mathrm{y}$ may have a high risk of extensive damage to their dentition, indicated by the development of conditions, such as hypodontia, microdontia, enamel hypoplasia, dental caries, and atypical crown or root formation $^{3,4)}$. In cases of arrested root development, short

Correspondence to: Dr. Jun Ohno, Oral Medicine Research Center, Fukuoka Dental College, 2-15-1 Tamura, Sawara-ku, Fukuoka, Fukuoka 8140193, Japan; Tel: +81928010411 (Ext 1684); Fax: +81928014909; E-mail: johno@ college.fdcnet.ac.jp
V-shaped roots and early closure of the apex are relatively common findings ${ }^{5}$. Root formation causes an overall increase in the tooth length to accompany intraosseous eruption during the process of emergence. Therefore, atypical root formation may cause disturbances in tooth eruption that severely impact the oral health quality of life in children receiving long-term chemotherapy.

Root and cementum formation are controlled by Hertwig's epithelial root sheath (HERS). The HERS, originating from an elongated apical region of the enamel organ, is composed of a bilayer epithelial structure between the dental papilla and dental follicle ${ }^{6}$; it grows apically and guides root formation, resulting in the determination of the size, shape, and number of the tooth's $\operatorname{root}^{7}$. Among the fate of HERS nearing completion of root formation, an induction of epithelial-mesenchymal transition (EMT) is responsible for participation in the cementum and periodontal ligament formation ${ }^{8,9}$. Our recent report suggests that impaired root formation induced by chemotherapy may result in early closure of apex via induced EMT of HERS cells ${ }^{10}$.

The EMT provides depolarization of the epithelial cells; loss of their cell-cell contacts; and gain of an elongated, fibroblast-like morphology. Functional implications of EMT include enhanced migratory capacity, invasiveness, elevated resistance to apoptosis, and substantially increased production of extracellular matrix components ${ }^{11)}$. In in vitro ex- 
periments, cells treated with transforming growth factor $\beta 1$ (TGF- $\beta 1$ ) can show the induction of marked EMT characteristics ${ }^{11,12)}$. A previous study has demonstrated that TGF- $\beta$ can induce EMT in HERS cell ${ }^{13)}$. The EMT process is regulated in part by Sonic hedgehog (Shh) signaling that corresponds to several biological processes in normal embryonic development and adult tissue homeostasis ${ }^{6}$. Once Shh binds to Patched (Ptch), an inhibition of a signal transducer Smoothened (Smo) is relieved, leading to the translocation of Glil proteins into the nucleus and the activation of Shh genes expressions ${ }^{14)}$. Activation of the Shh signaling pathway, including nuclear translocation of Gli1 can provide increased expression of snail, a major target of the Shh signaling pathway, as well as regulate EMT and fibroblast motility ${ }^{15}$. Although few studies have discussed the possibility of chemotherapy-induced EMT ${ }^{10,16)}$, whether cisplatin induces EMT in HERS cells remains unclear.

To the best of our knowledge, there has been no systematic study of the possible mechanisms of cisplatin that may induce EMT in HERS cells. Our previous study suggested that chemotherapy-induced EMT may provide the impaired root formation via an early closure of the apex $^{10)}$. In this study, we examined whether cisplatin could trigger EMT in HERS cells via the Shh/Glil signaling pathway.

\section{Cells}

\section{Materials and Methods}

The HERS cell line, HERS01a, was previously established ${ }^{17)}$. HERS01a cells were maintained in Dulbecco's Modified Eagle Medium: Nutrient Mixture F-12 (Fujifilm Wako Pure Chemical Corporation, Osaka, Japan) supplemented with B12 ((Invitrogen, Carlsbad, CA, USA), fibroblast growth factor-2 (FGF2, $10 \mathrm{ng} / \mathrm{ml}$, Fibroblast Spray, Kakei Pharmaceutical Co., Ltd., Tokyo, Japan), epidermal growth factor (EGF, $10 \mathrm{ng} /$ $\mathrm{ml}$, Pepro Tech, Rocky Hill, NJ, USA), and penicillin-streptomycin (100 $\mathrm{U} / \mathrm{ml}$ ) in a humidified atmosphere of $5 \% \mathrm{CO}_{2}$ and $37^{\circ} \mathrm{C}$. Upon reaching sub confluence, the cells were treated with medium containing various concentration of cisplatin $(0,0.5,1.0,5.0$, and $10 \mu \mathrm{M})$ for $24 \mathrm{~h}$. Furthermore, we also examined the effect of TGF- $\beta$ on the induction of EMT in the HERS cells. The cells were stimulated with medium containing 10 $\mathrm{ng} / \mathrm{ml}$ TGF- $\beta$ for $24 \mathrm{~h}$.

\section{Cell viability assay}

HERS cells were seeded in 96-well plates with $100 \mu 1$ of medium at a density of $1 \times 10^{4}$ cells $/ \mathrm{ml}$; they were incubated at $37^{\circ} \mathrm{C}$ overnight to allow the adherent cells to attach to the wells. The cultured cells were then incubated with cisplatin $(0-10 \mu \mathrm{M})$ for $24 \mathrm{~h}$. Thereafter, $10 \mu \mathrm{l}$ cell counting kit-8 (CCK-8; Dojindo Laboratories, Kumamoto, Japan) was added to each well, and the cultured cells were incubated at $37^{\circ} \mathrm{C}$ for 4 h. After incubation, the amount of formazan dye generated via cellular dehydrogenase activity was measured by absorbance at $480 \mathrm{~nm}$ as the detection wavelength and at $650 \mathrm{~nm}$ as the reference wavelength using a plate reader (Multiskan JX, Labsystems Oy, Helsinki, Finland).

\section{Western blot analysis}

The cells were lysed in cell lysis buffer (Cell Signaling Technology, Inc., Danvers, MA, USA) containing a 1x Protease/Phosphatase Inhibitor Cocktail (Cell Signaling Technology). The protein content was measured using a protein assay kit (Pierce, Hercules, CA, USA). Protein samples, with a protein marker (Precision Plus Protein Western C Standard; Bio-Rad Laboratories, Inc., Richmond, CA, USA), were separated on $12 \%$ Mini-Protean TGX gels (Bio-Rad) for $30 \mathrm{~min}$ at $200 \mathrm{~V}$. The separated gels were then transferred to a polyvinylidene fluoride membrane for $7 \mathrm{~min}$ with the Trans-Blot Turbo Transfer system (Bio-Rad) using Trans-Blot Transfer Packs. Western blots were processed on the iBind Western System (Life Technologies, Carlsbad, CA, USA) with anti-b-actin (Bio-Rad), anti-E-Cadherin (1:1,000; Cell Signaling Technology), anti-CK14 (1:1,000; Cell Signaling Technology), anti-vimentin (1:1,000; Cell Signaling Technology), anti-N-cadherin (1:1,000; Abcam, Cambridge, UK), sonic hedgehog (Shh, 1:1,000; Cell Signaling Technology), and horseradish peroxidase-conjugated anti-rabbit or -mouse secondary antibodies (Bio-Rad). The protein bands were developed with an enhanced chemiluminescence system (SignalFire Plus ECL Reagent, Cell Signaling Technology). The band density was quantified using the NIH ImageJ software.

\section{Immunocytochemical analysis}

The HERS cells were cultured on the 10-well glass slides and fixed with $4 \%$ paraformaldehyde for $10 \mathrm{~min}$ and washed in $0.3 \%$ Triton-X in phosphate buffered saline (PBS) for $10 \mathrm{~min}$. The cells were then incubated with anti-E-cadherin (1:200; Cell Signaling Technology), anti-vimentin (1:200; Cell Signaling Technology), anti-glioma-associated antigen-1 (Gli1, 1:100; Cell Signaling Technology), anti-snail (1:100; Cell Signaling Technology), and anti- $\gamma \mathrm{H} 2 \mathrm{AX}$ (1:100; Dojindo Laboratories), at $4{ }^{\circ} \mathrm{C}$ overnight. After washing with PBS, the cells were incubated with anti-rabbit IgG or anti-mouse $\operatorname{IgG}$ conjugated with Alexa Fluor 488 or 556 (1:400; Molecular Probe, Eugene, OR, USA) at room temperature for $45 \mathrm{~min}$. In order to visualize the nuclei, the cells were counterstained with 4, 6-diamidino-2-phenylindole (DAPI; Vector Laboratories, Inc., Burlingame, CA, USA). The percentages of positive cells were estimated under the $\times 40$ objective lens of a fluorescence microscope at 5 different sites of each slide glass.

\section{Blockade of the Shh/Gli1 signaling pathway}

Small-interfering RNA (siRNA) for Gli1 was used to inhibit the Shh/Gli1 pathway. The HERS01a cells were seeded in 24-well plates at a density of $5 \times 10^{4}$ cells/well and were transfected with siRNA for Gli1 (5'-AACUCCACAGGCAUACAGGAU-3') and a negative control siRNA (NC siRNA: 5'-AACGUACGCGGA AUACAACGA-3') were purchased from Hokkaido System Science Co., Ltd. (Sapporo, Japan) ${ }^{18}$. siRNA was introduced into the cells using HilyMax (Dojindo Laboratories). Twenty-four hours thereafter, the cells were stimulated with or without cisplatin. After treatment with or without cisplatin for $24 \mathrm{~h}$, those cells were stained with antibodies for downstream target factors in the Shh/Glil signaling pathway. All the siRNA experiments were performed in triplicate.

\section{Statistical analyses}

All the statistical analyses were performed with EZR (Saitama Medical Center, Jichi Medical University, Saitama, Japan), a graphical user interface for R (The R Foundation for Statistical Computing, Vienna, Austria). More precisely, it is a modified version of the $\mathrm{R}$ commander designed to add statistical functions frequently used in biostatistics. The analyses were performed using one-way analysis of variance and Tukey multiple comparison test to determine the statistical differences among the samples. Data are presented as the mean \pm standard deviation (SD) values, and $p$ values $<0.05$ were considered to indicate statistical significance.

\section{Results}

Low concentration of cisplatin treatment does not decrease the cell viability

Cell viability was detected using the CCK-8 assay. The HERS cells 


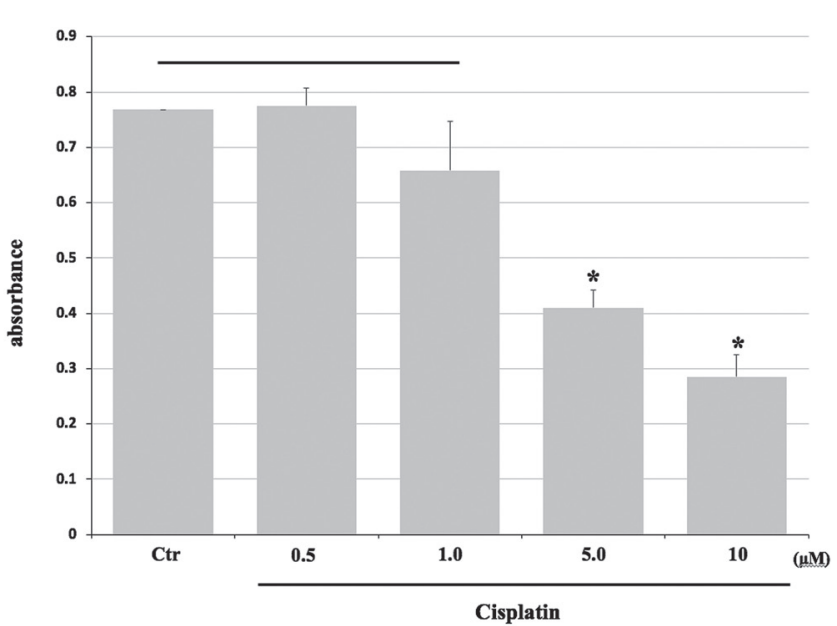

Figure 1. Effect of cisplatin on cell viability. Cell viability of the HERS cells treated with 0 (Ctr, control) $-10 \mu \mathrm{M}$ cisplatin for $24 \mathrm{~h}$ using the Cell Counting Kit-8 assay. There was no significant difference in the groups of gray columns jointed to horizontal bar. *, significant difference at $p<0.001$ compared with 5- and 10- $\mu \mathrm{M}$ cisplatin-treated groups.

were treated with 0 (as a control), $0.5,1.0,5.0$, and $10 \mu \mathrm{M}$ cisplatin for $24 \mathrm{~h}$ to estimate the cell viability. As shown in Fig. 1, low-concentration treatment groups $(0.5$ and $1.0 \mu \mathrm{M})$ showed no significant reduction in the cell viability as compared to the control group $(p>0.2)$, while the other groups $(5.0 \mu \mathrm{M}$ and $10 \mu \mathrm{M})$ notably reduced the viability of the HERS cells $(p<0.001)$ in a dose-dependent manner.

\section{Low concentration of cisplatin treatment can induce DNA damage in HERS cells}

Cisplatin kills cancer cells by damaging their DNA. During DNA damage by stimulus, $\gamma \mathrm{H} 2 \mathrm{AX}$ is produced via the phosphorylation of H2AX. We performed immunocytochemical detection of nuclear $\gamma \mathrm{H} 2 \mathrm{AX}$ to determine whether cisplatin can induce DNA damage in the HERS cells (Fig. 2A). Most untreated HERS cells showed only few positive cells $(14.5 \% \pm 2.8 \%)$. Promotion of nuclear $\gamma \mathrm{H} 2 \mathrm{AX}$ staining was gradually increased in the HERS cells treated with $0.5 \mu \mathrm{M}$ and 1.0 $\mu \mathrm{M}$ cisplatin $(43.2 \% \pm 12.0 \%$ and $57.2 \% \pm 5.4 \%$, respectively). There was a significant difference in the proportion of nuclear staining between untreated ( $\mathrm{Ctr}$ ) and $0.5-\mu \mathrm{M}$ cisplatin-treated cells $(p=0.012)$ (Fig. 2B). These findings indicate that $0.5 \mu \mathrm{M}$ cisplatin treatment does not reduce the cell viability significantly; however, this concentration of cisplatin can induce DNA damage in the HERS cells. Thus, we selected this concentration as the effective concentration for performing further research.

\section{Low concentration of cisplatin can induce EMT in the HERS cells}

In order to investigate whether cisplatin treatment induces EMT in the HERS cells, we first examined that EMT of HERS cells was induced via stimulation of TGF- $\beta$, known as an EMT inducer. After stimulation by TGF- $\beta$, the cells acquired an elongated and mesenchymal-like morphology with loss of cell-cell contact, while the control cells remained a cobblestone morphology as epithelial characteristics (Fig. 3A). Western blot analysis showed that the protein level of both, E-cadherin and CK14 was decreased. In contrast, the cells treated with TGF- $\beta$ showed increased expression of $\mathrm{N}$-cadherin, as compared to that of the controls (Fig. 3B). Intensity of vimentin expression in TGF- $\beta$-treated cells was similar to that of the control cells (Fig. 3B).

Then, we examined whether low concentration of cisplatin $(0.5 \mu \mathrm{M}$ and $1.0 \mu \mathrm{M}$ ) could induce a morphological change characteristic of EMT. The HERS cells without cisplatin exhibited a cobblestone-epithelial morphology. However, the cells stimulated by cisplatin showed a mesenchymal-like morphology with an elongated shape and loss of cellcell contact (Fig. 3C). In the western blot analysis, downregulated expression of epithelial markers (E-cadherin and CK14) in the cells treated with $0.5 \mu \mathrm{M}$ cisplatin was noted. Cisplatin-treated cells showed increased expression of $\mathrm{N}$-cadherin in a dose-dependent manner, as compared to the control cells (Fig. 3D). Vimentin expression was increased in the cells treated with $0.5 \mu \mathrm{M}$ cisplatin, whereas in the $1.0 \mu \mathrm{M}$ cisplatin-treated cells vimentin expression was suppressed (Fig. 3D). These findings indicated that cisplatin-induced EMT characteristics in the HERS cells.
$\mathbf{A}$

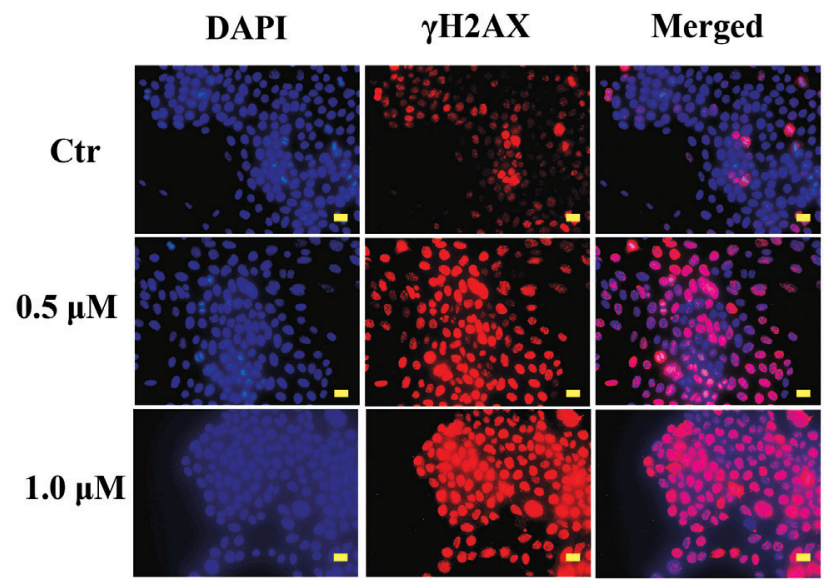

B

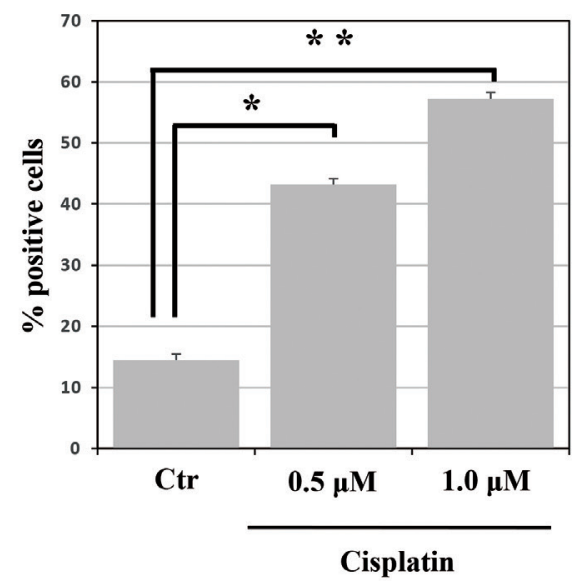

Figure 2. Increased promotion of cisplatin-treated HERS cells with nuclear $\gamma \mathrm{H} 2 \mathrm{AX}$ staining. HERS cells treated with $(0.5$ and $1.0 \mu \mathrm{M})$ and without cisplatin were labeled for $\gamma \mathrm{H} 2 \mathrm{AX}$ (red). The nuclei were stained with DAPI (blue). A: Representative immunofluorescence images of nuclear $\gamma \mathrm{H} 2 \mathrm{AX}$ staining. Bars $=20 \mu \mathrm{m}$. B: Proportion (\%) of HERS cells with nuclear $\gamma \mathrm{H} 2 \mathrm{AX}$ staining as mean \pm standard deviation (SD) values $(\mathrm{n}=3) . *{ }^{* *}$, Significant differences at $p<0.05, P<0.001$, respectively, compared with Ctr. 
A

\section{TGF-ק1}

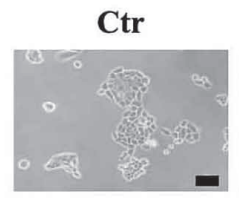

B

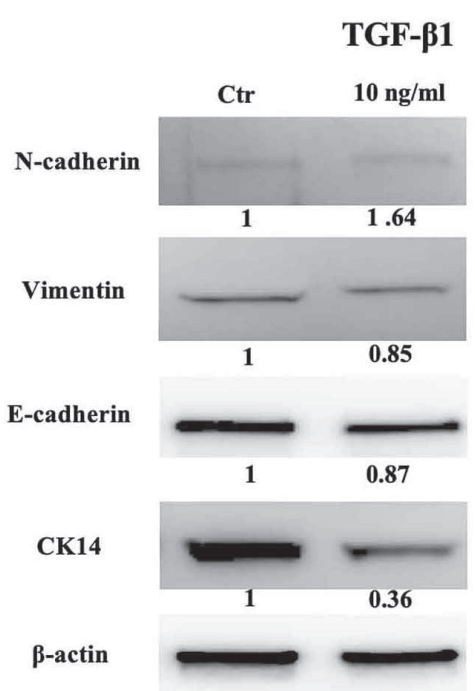

C

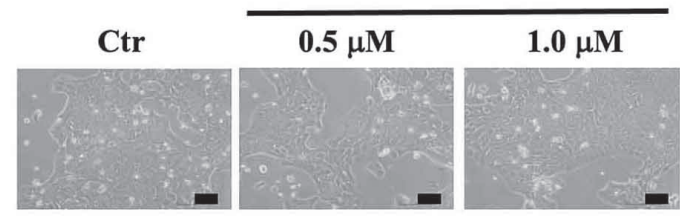

D

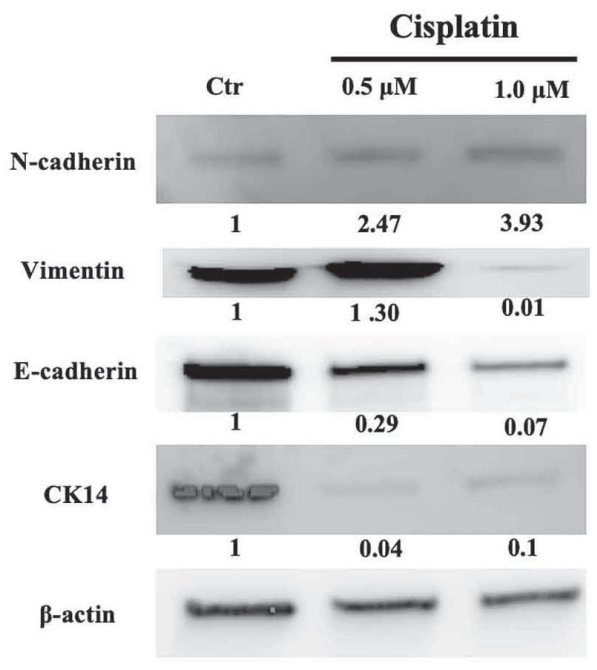

Figure 3. TGF- $\beta$ and cisplatin induces EMT in the HERS cells. A: Representative phase contrast images of a morphological phenotype in the HERS cells treated with or without TGF- $\beta$. B: HERS cells were stimulated with TGF- $\beta(10 \mathrm{ng} / \mathrm{ml})$ or in complete medium for $24 \mathrm{~h}$. Representative western blots using antibodies against N-cadherin, vimentin, E-cadherin, and CK14. C: Representative phase contrast images of EMT induction in HERS cells treated with or without cisplatin. D: The HERS cells were treated with cisplatin $(0.5$ and $1.0 \mu \mathrm{M})$ or in complete medium for $24 \mathrm{~h}$. Representative western blots using antibodies against N-cadherin, vimentin, E-cadherin, and CK14. Ctr, control. Scale bars: $20 \mu \mathrm{m}$.

A

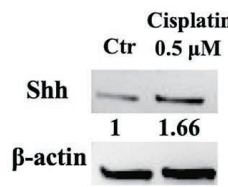

B
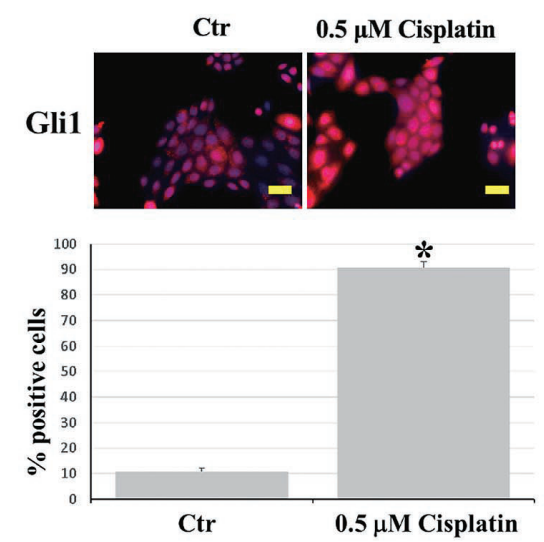

C
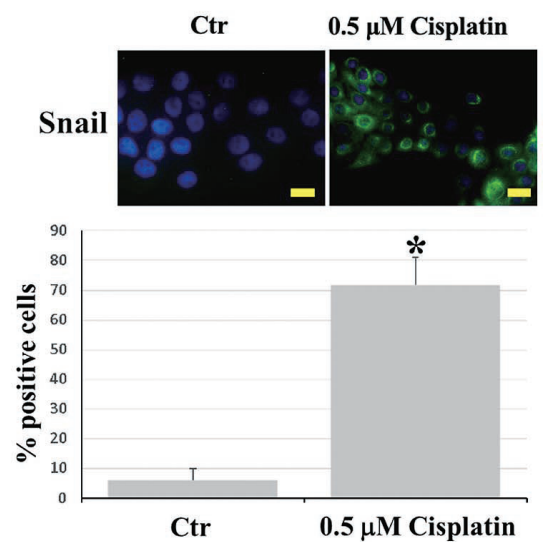

Figure 4. Shh/Gli1 pathway in the HERS cells treated with $0.5 \mu \mathrm{M}$ cisplatin. A: Representative western blot using antibody against Shh. B and C: Immunofluorescence images of nuclear translocation of Gli1 (B; red) and cytoplasmic staining with snail (C; green) in HERS cells treated with or without cisplatin. Nuclei were stained with DAPI. The graphs show quantified data of the percentages of positive cells stained with anti-Gli1 and snail as mean \pm standard deviation $(\mathrm{SD})(\mathrm{n}=3) .{ }^{*}$, significant difference at $p<0.001$ with $\mathrm{Ctr}$. Ctr, control; NC siRNA, negative control siRNA. Scale bars: $20 \mu \mathrm{m}$. 
Hanako Ishii et al.: Cisplatin-Induced Epithelial-Mesenchymal Transition in HERS Cells

In the HERS cells, $0.5 \mu \mathrm{M}$ cisplatin induces enhancement of the Shh/ Gli1 pathway

We examined the expressions of Shh and Gli1 in HERS cells treated with or without $0.5 \mu \mathrm{M}$ cisplatin to investigate whether Shh/Gli1 pathway is related to cisplatin-induced EMT. As indicated by the results of the western blotting analysis (Fig. 4A), the cells exposed to $0.5 \mu \mathrm{M}$ cisplatin for $24 \mathrm{~h}$ showed increased Shh expression as compared to that in the control cells. The nuclear translocation of Gli1 is a key inducer of $\mathrm{EMT}^{19)}$. Then, we examined the immunocytochemical detection of Gli1 in HERS cells treated with or without $0.5 \mu \mathrm{M}$ cisplatin. The nuclear localization and accumulation of Gli1 was observed in HERS cells treated with $0.5 \mu \mathrm{M}$ cisplatin (Fig. 4B). The percentages of nuclear positive cells were much higher in the $0.5-\mu \mathrm{M}$ cisplatin-treated cells than in the control cells $(90.8 \% \pm 2.2 \%$ vs. $10.9 \% \pm 1.4 \%, p<0.001$; Fig. 4B). Furthermore, snail is a well-known target of the Shh/Gli1 signaling ${ }^{18)}$. We finally performed immunocytochemical detection of snail in HERS cells treated with or without $0.5 \mu \mathrm{M}$ cisplatin. Cisplatin-treated cells showed cytoplasmic reaction in snail, while no staining was observed in the untreated cells (Fig. 4C). The percentages of snail-positive cells were higher in the $0.5-\mu \mathrm{M}$ cisplatin-treated cells than in the control cells $(71.8 \% \pm 9.2 \%$ vs. $6.0 \% \pm 4.0 \%, p<0.001$; Fig. 4 C). These results suggest that cisplatin-induced EMT may be partially regulated by the Shh/ Gli1 signaling pathway.

\section{Suppression of Gli1 signaling reduces cisplatin-induced EMT in HERS cells}

In order to examine whether Gli1 signaling mediates $0.5 \mu \mathrm{M}$ cisplatin-induced EMT, siRNA was used for silencing Gli1. Transfection of Gli1 siRNA into the HERS cells attenuated immunocytochemical expression of Glil protein in these cells (Fig. 5A). The transfected cells were then treated with $0.5 \mu \mathrm{M}$ cisplatin. Compared to transfection with a negative control siRNA, immunofluorescence images and semi-quantified graphs of the percentage of positive cells showed that transfection with Gli1-specific siRNA significantly decreased the EMT process, as demonstrated by the lower expression of snail and vimentin and the increased expression of E-cadherin (Fig. 5A, B). These findings suggest that inhibition of Shh/Gli1 signaling effectively suppressed the EMT process in vitro.

A
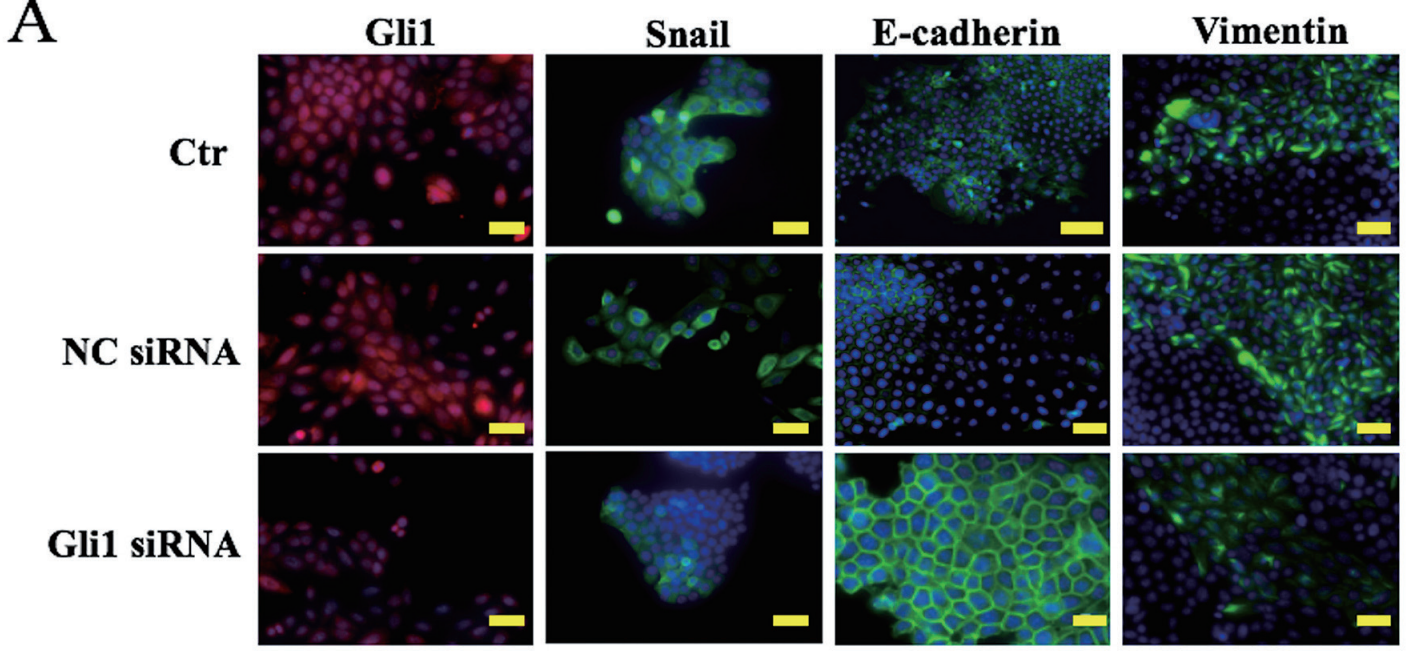

B
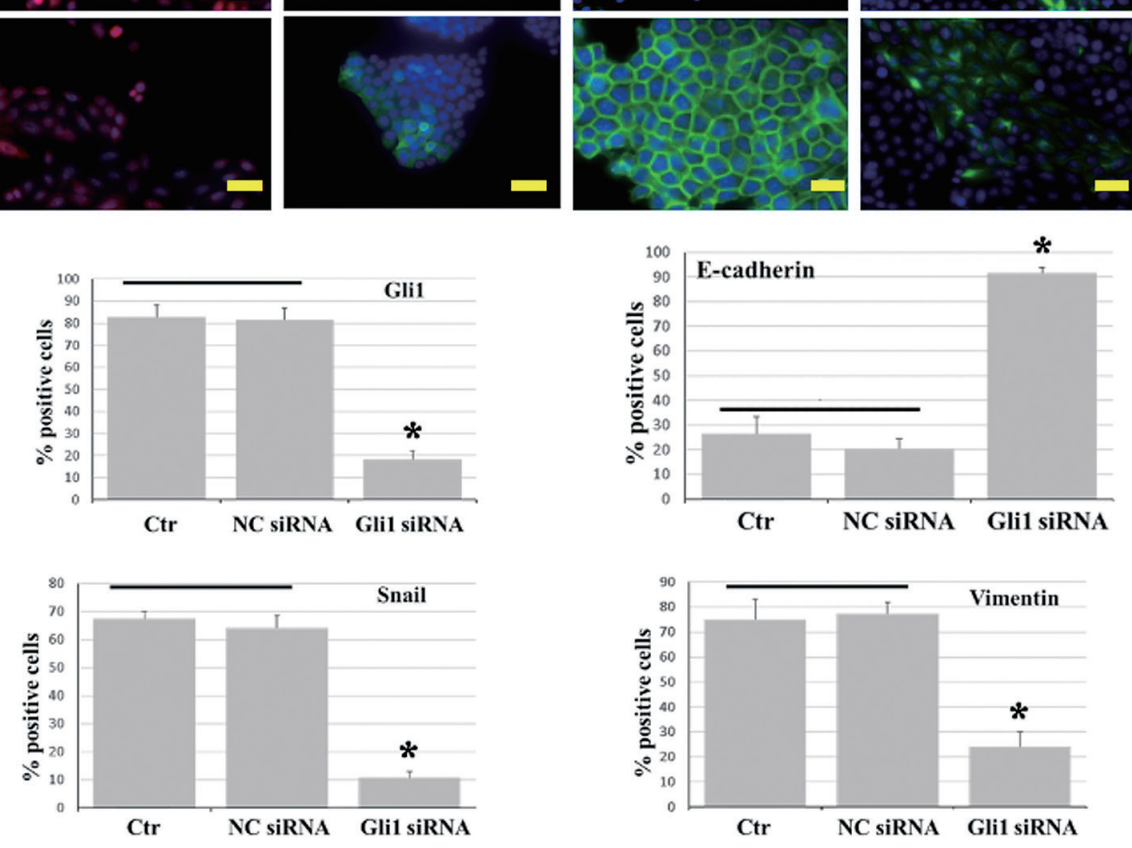

Figure 5. Reduction of cisplatin-mediated EMT in the HERS cells by blockade of Gli1 signaling. The cells were stimulated with $0.5 \mu \mathrm{M}$ cisplatin in the presence of a Glil siRNA or NC siRNA as indicated. A: Effect of the silencing of Glil using siRNA was assessed with immunofluorescence staining. The cells were stained with anti-Glil (red), snail (green), E-cadherin (green), and vimentin (green) antibodies. Nuclei were stained with DAPI (blue). Bars $=20 \mu \mathrm{m}$. B: Graphs show semi-quantified data of the percentages of positive cells stained with anti-Glil, snail, E-cadherin, and vimentin as mean \pm standard deviation $(\mathrm{SD})(\mathrm{n}=3) .{ }^{*}$, significant difference at $p<0.001$ with Ctr and NC siRNA groups. Ctr, control; NC siRNA, negative control siRNA. 


\section{Discussion}

Our previous findings suggested that the administration of anticancer drug can cause arrested root development of the molars in mice via an induced EMT-like phenomenon ${ }^{10}$. Here we demonstrate that low concentration of cisplatin promotes EMT in the HERS cells via the activation of Shh/Gli1 signaling pathway by induction of DNA damage. Our conclusion is supported by the following observations: (a) low concentration of cisplatin $(0.5 \mu \mathrm{M})$ induces DNA damage; however, this concentration does not reduce the cell viability; (b) cisplatin-induced EMT is examined on the basis of downregulated and up-regulated expression of epithelial and mesenchymal markers, respectively; (c) the cisplatin-treated HERS cells showed increased Shh and Gli1 expressions; (d) Transfection of Gli1 siRNA into the HERS cells reveals a significant suppression of cisplatin-induced EMT.

Low concentration of cisplatin is critical for inducing EMT in the HERS cells. Our results from CCK-8 assay and $\gamma \mathrm{H} 2 \mathrm{AX}$ staining showed that $0.5 \mu \mathrm{M}$ cisplatin treatment showed no reduction of the cell viability and no induction of cellular senescence. By $\gamma \mathrm{H} 2 \mathrm{AX}$ staining, DNA damage was observed in HERS cells treated with $0.5 \mu \mathrm{M}$ cisplatin, but those cells did not reduce the viability of them. Cisplatin is an important anticancer drug that kills cancer cells by damaging their DNA; the formation of cisplatin-DNA adducts interferes with DNA replication and transcription $^{20,21)}$. H2AX is a key factor in the process of repair for damaged DNA and is recruited to the damaged sites. In the presence of DNA damage, H2AX is phosphorylated on the 139th serine residue, termed $\gamma \mathrm{H} 2 \mathrm{AX}$. Of the methods for detecting DNA damage of the cells, such as neutral elution, pulse field electrophoresis, and comet assays, $\gamma \mathrm{H} 2 \mathrm{AX}$ assay is a particularly precise method ${ }^{22)}$. Although nuclear $\gamma \mathrm{H} 2 \mathrm{AX}$ staining was found in all the cisplatin-treated cells, irrespective of cisplatin concentration, no reduction in cell viability was obtained only in the HERS cells treated with low concentration of cisplatin $(0.5 \mu \mathrm{M}$ and 1.0 $\mu \mathrm{M})$. Our results that $0.5 \mu \mathrm{M}$ cisplatin treatment triggers marked EMT in HERS cells suggest that EMT induction may depend on the degree of DNA damage caused by cisplatin treatment. Activation of a DNA damage response, including formation of DNA damage foci containing $\gamma \mathrm{H} 2 \mathrm{AX}$ staining at persistent DNA strand breaks is the major trigger for cell senescence ${ }^{23)}$. HERS cells treated with $0.5 \mu \mathrm{M}$ cisplatin maintained a negative reaction with SA- $\beta$-Gal staining, a marker for cellular senescence, although the cells treated with other concentrations of cisplatin showed marked cytoplasmic staining with SA- $\beta$-Gal (data not shown). Therefore, the pre-senescence status of $0.5 \mu \mathrm{M}$ cisplatin-treated cells could be susceptible for EMT induction in HERS cells.

In the present study, EMT induction was examined with a low concentration of cisplatin in addition to TGF- $\beta$ treatment. TGF- $\beta$-induced EMT is a well-known and fundamental process in organogenesis and cancer progression $^{24,25)}$. The HERS cells stimulated by cisplatin or TGF$\beta$ exhibited morphological and biochemical features of EMT; western blot assay showed that the expression of epithelial markers was decreased, while the expression of mesenchymal markers was increased, compared with than in controls. The exposure of the HERS cells to low concentration of cisplatin facilitated EMT. These findings of TGF- $\beta$-induced EMT in the HERS cells are in line with those reported by a previous study ${ }^{13)}$. As TGF- $\beta$-induced EMT appears to be related to the completion of root formation, chemotherapy-induced EMT may lead to the eruption disturbances via early closure of the apex in children undergoing chemotherapy.

Western blot assay and immunofluorescence staining revealed that HERS cells treated with $0.5 \mu \mathrm{M}$ cisplatin showed enhanced activity of the Shh signaling pathway. The Shh pathway plays an important role during the processes of embryonic development and tumorigenesis. In the present study, we first detected an increase in the protein expression of Shh in HERS cells treated with $0.5 \mu \mathrm{M}$ cisplatin. With immunofluorescence staining, we found that the nuclear translocation of Gli1 was significantly enhanced in the HERS cells exposed to cisplatin than in the controls. The nuclear localization and accumulation of Glil is essential for its transcriptional activity. Enhanced translocation of Gli1, a target of Shh, indicates the activation of the Shh signaling pathway ${ }^{14)}$. These findings suggest that the expression of Shh/Glil unregulated in response to cisplatin treatment. In our immunocytochemical staining, the number of Snail-expressed cells were significantly increased in the HERS cells treated with cisplatin. A recent study has emphasized the crucial roles of the transcription factor Snail during the EMT process ${ }^{16)}$. Snail is also the target of the Shh/Gli1 signaling pathway ${ }^{14)}$. Based on these findings, we suggest that cisplatin-induced EMT may be partially mediated by the Shh/Gli1 signaling pathway.

Gene silencing of Gli1 demonstrated that the activity of Shh/Gli1 signaling pathway can promote cisplatin-induced EMT in the HERS cells. Immunofluorescence assay showed that the percentages of E-cadherin-positive cells were decreased in cisplatin-treated cells in the presence of Glil siRNA than in the presence of negative control siRNA. In contrast, the percentages of vimentin-positive cells were increased in the Gli1 siRNA group. Furthermore, following the blockade of Gli1 expression by siRNA, cisplatin-treated HERS cells had lower cytoplasmic Snail expression. Based on these data, we proposed that the treatment with cisplatin activated Shh/Gli1 signaling pathway, which unregulated the nuclear translocation of Gli1 and in turn increased Snail expression, finally resulting in induction of EMT.

The present study has certain limitations, especially in terms of the lack of direct evidence regarding whether the blockade of Shh signaling can suppress the activity of the cisplatin-induced EMT. Immunocytochemical assay showed that silencing of Gli1, a target of Shh signaling inhibited the cisplatin-induced EMT. Further studies are required to examine the vital role of Shh in the cisplatin-induced EMT using pretreatment of cyclopamine ${ }^{18)}$, an Shh inhibitor. In this study, most data were obtained via immunofluorescence staining. We believe that protein expression data must be acquired using western blotting and used in the experiments of silencing or blockade of genes in further studies.

Thus, our findings provide additional information about the effect of anticancer drugs on EMT promotion in HERS cells. We demonstrated that low concentrations of cisplatin promote EMT in HERS cells via the activation of the Shh/Gli1 signaling pathway by inducing DNA damage.

\section{Acknowledgement}

We would like to thank Enago (Academic Proofreading Service; www.enago.jp) for the English language review. This work was supported by JSPS KAKENHI (grant number: 18K09567 to JO) and the Private University Research Branding Project.

\section{Conflict of Interest}

The authors have no competing interest to declare.

\section{References}

1. Ruggiero A, Trombatone G, Triarico S, Arena R, Ferrara P, Scalzone M, Pierri F and Riccardi R. Platinum compounds in children with cancer: toxicity and clinical management. Anticancer Drugs 24: 1007-1019, 2013

2. McKeage MJ. New-generation platinum drugs in the treatment of cisplatin-resistant cancers. Expert Opin lnvestig Drugs 14: 1033- 
1046,2005

3. Goho C. Chemoradiation therapy: effect on dental development. Journal Pediatr Dent 15: 6-12, 1993

4. Cubukcu CE, Sevinir B and Ercan I. Disturbed dental development of permanent teeth in children with solid tumors and lymphomas. Pediatr Blood Cancer 58: 80-84, 2012

5. Purdell-Lewis DJ, Stalman MS, Leeuw JA, Humphrey GB and Kalsbeek H. Long term results of chemotherapy on the developing dentition: caries risk and developmental aspects. Community Dent Oral Epidemiol 16: 68-71, 1988

6. Orban BJ. Orban's Oral histology and embryology. Mosby, St. Louis, 1980

7. Cate ART. The role of epithelium in the development, structure and function of the tissues of tooth support. Journal Oral Dis 2: 55-52, 1996

8. Huang X, Bringas P Jr, Slavkin HC and Chai Y. Fate of HERS during tooth root development. Dev Biol 334: 22-30, 2009

9. Luan X, Ito Y and Diekwisch TG. Evolution and development of Hertwig's epithelial root sheath. Dev Dyn 235: 1167-1180, 2006

10. Matsuo S, Toda-Nakamura M, Oka K, Kajiya H, Ogata K, Ishii H, Ozaki $\mathrm{M}$ and Ohno J. Cyclophosphamide promotes arrested development of the dental root in mice. J Hard Tissue Biol 29: 63-70, 2020

11. Kalluri R and Weinberg RA. The basics of epithelial-mesenchymal transition. J Clin Invest 119: 1420-1428, 2009

12. Scheel C and Weinberg RA. Phenotypic plasticity and epithelial-mesenchymal transitions in cancer - and normal stem cells? Int J Cancer 129: 2310-2314, 2011

13. Itaya S, Oka K, Ogata K, Tamura S, Kira-Tatsuoka M, Fujiwara N, Otsu K, Tsuruga E, Ozaki M and Harada H. Hertwig's epithelial root sheath cells contribute to formation of periodontal ligament through epithelial-mesenchymal transition by TGF-beta. Biomed Res 38: 61-69, 2017

14. Rimkus TK, Carpenter RL, Qasem S, Chan M and Lo HW. Targeting the sonic hedgehog signaling pathway: review of smoothened and GLI inhibitors. Cancers 8: E22, 2016

15. Thiery JP, Acloque H, Huang RYJ and Nieto MA. Epithelial-mesenchymal transitions in development and disease. Cell 139: 871-890, 2009

16. Fang S, Yu L, Mei H, Yang J, Gao T, Cheng A, Guo W, Xia K and Liu G. Cisplatin promotes mesenchymal-like characteristics in osteosarcoma through snail. Oncoll Lett 12: 5007-5014, 2016

17. Akimoto T, Fujiwara N, Kagiya T, Otsu K, 1shizeki K and Harada H. Establishment of Hertwig's epithelial root sheath cell line from cells involved in epithelial-mesenchymal transition. Biochem Biophys Res Commun 404: 308-312, 2011

18. Zou Y, Song W, Zhou L, Mao Y and Hong W. House dust mite induces sonic hedgehog signaling that mediates epithelialmesenchymal transition in human bronchial epithelial cells. Mol Med Rep 20: 4674-4682, 2016

19. Grindley JC, Bellusci S, Perkins D and Hogan BLM. Evidence for the involvement of the Gli gene family in embryonic mouse lung development. Dev Biol 188: 337-348, 2016

20. Hu J, Lieb JD, Sancar A and Adar S. Cisplatin DNA damage and repair maps of the human genome at single- nucleotide resolution. Proc Natl Acad Sci USA 113: 11507-11512, 2016

21. Basu A and Karishnamurthy S. Cellular responses to cisplatin-induced DNA damage. J Nucleic Acids 2010: 201367, 2010

22. Kuo LJ and Yang LX. Gamma-H2AX - a novel biomarker for DNA double-strand breaks. In Vivo 22: 305-309, 2008

23. Wang C, Jurk D, Maddick M, Nelson G, Martin-Ruiz C and von Zglinicki T. DNA damage response and cellular senescencein tissues of aging mice. Aging Cell 8: 311-323, 2009

24. Katsuno Y, Lamouille S and Derynck R. TGF-beta signaling and epithelial-mesenchymal transition in cancer progression. Curr Opin Oncol 25: 76-84, 2013

25. Wang Y, Shi J, Chai K, Ying X and Zhou BP. The role of snail in EMT and tumorigenesis. Curr Cancer Drug Targets 13: 963-972, 2013 
J.Hard Tissue Biology Vol. 30(2): 115-122, 2021 